\title{
Produção familiar do camarão Litopenaeus vannamei (Boone, 1931): VIABILIDADE E QUALIDADE, VISANDO À SUSTENTABILIDADE
}

\author{
Nathiene Patrícia Ferreira Amaral Rolim¹, Ilda Antonieta Salata Toscano², Gil Dutra Furtado ${ }^{3}$, Flávia \\ Oliveira Paulino ${ }^{4}$, Maria Cristina Basílio Crispim ${ }^{5}$
}

1 Mestre pelo Programa de Pós-graduação em Desenvolvimento e Meio Ambiente, Universidade Federal da Paraíba.

2, 3, 4 e 5 Docentes do Programa de Pós-graduação em Desenvolvimento e Meio Ambiente, Universidade Federal da Paraíba.

Recebido em 10 de julho de 2017. Aceito em 23 de março de 2018. Publicado em 28 de dezembro de 2018.

Resumo - Com vistas a atender a demanda por alimentos de origem marinha, com qualidade e valor nutricional adequado, o emprego de técnicas de cultivo de camarões em tanques associadas à produção de ração doméstica pode ser uma alternativa viável para a obtenção de um produto de boa qualidade, ambientalmente responsável e com valor comercial agregado. A proposta deste trabalho foi avaliar o cultivo do camarão Litopenaeus vannamei (Boone, 1931) nos moldes da produção familiar, alimentados com ração doméstica, produzida a partir de resíduos de alimentos e biofilme, e ração industrial. Os resultados foram confrontados entre si para avaliação do tratamento proposto e a qualidade nutricional dos animais cultivados. Os dados indicaram que não houve diferença significativa entre os cultivos, no que se refere à composição nutricional dos animais, havendo diferença significativa entre os camarões alimentados com biofilme entre os alimentados sem biofilme. Portanto, pode-se entender que a ração doméstica, associada à oferta regular de biofilme, é uma alternativa aceitável para a substituição da ração industrial no cultivo familiar de camarões, devido ao baixo custo na produção, ausência de aditivos e conservantes, aproveitamento de resíduos gerados pela comunidade, além da sustentabilidade socioambiental da atividade.

Palavras-chave: camarão orgânico; Carcinicultura; tecnologia alternativa.

FAMILY PRODUCTION OF SHRIMP LITOPENAEUS VANNAMEI (BOONE, 1931): VIABILITY AND QUALITY, AIMING AT SUSTAINABILITY

Abstract - In order to meet the demand for marine food with adequate quality and nutritional value, the use of shrimp culture techniques in tanks associated with domestic feed production may be a viable alternative for obtaining a good product quality, environmentally responsible and with added commercial value. The proposal of this work was to evaluate the cultivation of shrimp Litopenaeus vannamei (Boone, 1931) in the form of family production, fed with domestic feed, produced from food waste and biofilm, and industrial feed. The results were compared among each other to evaluate the proposed treatment and the nutritional quality of the cultivated animals. The data indicated that there was no significant difference between the cultures, regarding the nutritional composition of the animals, and there was a significant difference between the shrimp fed with biofilm among those fed without biofilm. Therefore, it can be understood that the domestic ration, together with the regular supply of biofilm, is an acceptable alternative for the substitution of industrial ration in the family shrimp farming, due to the low cost of production, absence of additives and preservatives, use of residues generated by the community, as well as the socio-environmental sustainability of the activity.

KEYWORDS: ORGANIC SHRIMP; SHRIMP FARMING; ALTERNATIVE TECHNOLOGY. 
Producción familiar del camarón Litopenaeus vannamei (Boone, 1931): viabilidad y Calidad, con miras a la SOSTENIBILIDAD

Resumen - Con vistas a atender a la demanda de alimentos de origen marino, con calidad y valor nutricional adecuado, el empleo de técnicas de cultivo de camarones en estanques asociados a la producción de alimento natural de consumo humano puede ser una alternativa viable para la obtención de un producto de buena calidad, ambientalmente responsable y con valor comercial agregado. La propuesta de este trabajo fue evaluar el cultivo del camarón Litopenaeus vannamei (Boone, 1931) en los moldes de la producción familiar, alimentados con alimento doméstico, producida a partir de residuos de alimentos y biopelícula, y alimento industrial. Los resultados se confrontaron entre sí para evaluar el tratamiento propuesto y la calidad nutricional de los animales cultivados. Los datos indicaron que no hubo diferencia significativa entre los cultivos, en lo que se refiere a la composición nutricional de los animales, con diferencia significativa entre los camarones alimentados con biopelícula y los alimentados sin biopelícula. Por lo tanto, se puede entender que la alimentación doméstica, asociada a la oferta regular de biopelícula, es una alternativa aceptable para la sustitución de la alimentación industrial en el cultivo familiar de camarones, debido al bajo costo en la producción, ausencia de aditivos y conservantes, aprovechamiento de residuos generados por la comunidad, además de la sostenibilidad socioambiental de la actividad.

Palabras clave: camarón orgánico; camarones; tecnología alternativa.

\section{INTRODUÇÃO}

O crescimento populacional e a elevada necessidade de obter maiores quantidades de alimentos de origem marinha têm incentivado o desenvolvimento tecnológico para potencializar a produção na aquicultura. Dentre os meios de produção existentes, pode-se destacar a carcinicultura como uma das atividades da aquicultura que mais cresce no Brasil, principalmente na região Nordeste. A expansão dessa atividade pode ser justificada pelo alto valor do produto, tanto no mercado interno, como no externo, incentivo de bancos públicos, bem como a disponibilidade de terras "baratas" para a instalação de fazendas de cultivo (Dias et al. 2012).

No Brasil, o camarão mais cultivado nos moldes da carcinicultura é o da espécie exótica Litopenaeus vannamei que demonstrou alta capacidade de adaptação aos ecossistemas, clima e temperatura da região, obtendo índices de produtividade e rentabilidade superiores aos das espécies nativas (Sebrae 2008).

Para o cultivo de animais aquáticos, requer-se a proximidade com recursos hídricos, especificamente a carcinicultura que se instala, preferencialmente nos estuários. Tal proximidade contribuiu para a ocorrência de sérios impactos ambientais, como a deposição de resíduos oriundos dessa atividade, provocando sérias alterações nos ecossistemas aquáticos. Nessa perspectiva, a expansão da carcinicultura tem causado grande preocupação por parte dos órgãos ambientais seja pelos impactos negativos da atividade causados durante a sua instalação, pelo desmatamento, geralmente nos ecossistemas manguezais, seja pela frágil legislação específica que garanta os benefícios dessa atividade, sem degradar o meio ambiente (Figueirêdo 2006) e principalmente pela falta de fiscalização, que não efetiva o cumprimento da lei.

No intuito de expandir a atividade e obter maiores rendimentos são fornecidas rações alimentares, para os camarões marinhos, de modo que sejam disponibilizados os nutrientes necessários para o crescimento e desenvolvimento, com peso adequado e em tempo hábil, para a realização da despesca. Todavia, o fornecimento das rações comerciais de baixa qualidade tem levantado alguns questionamentos sobre a contaminação dos animais que pode ocorrer devido ao uso de ingredientes de baixa qualidade na formulação dessas rações, constituindo fontes potenciais de contaminação. Como por exemplo, as farinhas de peixes de baixa qualidade, que são um dos principais ingredientes das rações industriais (Alves-Costa e Costa 2004; Kasper 2007; Coutinho 2008). Embora seja um importante vetor de contaminação dos animais, cultivados nos moldes da cadeia produtiva, o controle na 
qualidade de água e o uso de rações de boa qualidade, podem contribuir para uma melhor qualidade do produto final.

Neste sentido, é imprescindível que as rações disponibilizadas possuam não apenas dietas eficientes, mas custos efetivos e ambientalmente sustentáveis, visto que o emprego inadequado das rações pode comprometer tanto o desenvolvimento animal como o meio ambiente (Furtado et al. 2010). Com vistas a atender à demanda por alimentos de origem marinha, com qualidade e valor nutricional adequado, a carcinicultura vem desenvolvendo várias tecnologias para potencializar a produção, por meio da redução dos custos e dos impactos ambientais da atividade. O cultivo destes animais em tanques de polipropileno, com emprego da técnica dos bioflocos, com adição de probióticos, com ou sem renovação de água, são alguns exemplos de tecnologias alternativas que objetivam o desenvolvimento sustentável do segmento (Figuerêdo 2006).

Desta forma é imprescindível o investimento em tecnologias voltadas para a carcinicultura sustentável, otimizando toda a cadeia produtiva desde a instalação até o momento da despesca. As técnicas de cultivo podem ser diferenciadas conforme o alimento fornecido (rações industrializadas, alimentos naturais presentes nos corpos hídricos ou a combinação de ambos) e pela frequência da renovação de água do cultivo (Capistrano Sobrinho 2011).

Nessa esteira, o cultivo de camarões em caixas d'água, ou viveiros fora do ambiente, em sistema fechado com volume de água reduzido, mantido em recirculação, com a oferta de alimentação natural ou artificial constitui uma tecnologia viável na promoção da expansão da atividade. Isso devido aos benefícios causados ao meio ambiente, uma vez que favorece a sua instalação fora dos locais tradicionais da carcinicultura reduzindo a emissão de efluentes nas áreas de mangue, visto que constitui uma das importantes fontes de contaminação ambiental, além de poder promover a distribuição de renda entre grupos sociais que historicamente foram excluídos do processo de desenvolvimento da aquicultura (Rolim 2015).

O Presente estudo objetivou avaliar o cultivo dos camarões orgânicos nos moldes da agricultura familiar, bem como a viabilidade e qualidade do produto final, apontando ainda os benefícios da utilização desta tecnologia para a redução dos impactos ambientais específicos da carcinicultura, e dos sociais através da promoção ao acesso e distribuição de renda entre os pescadores.

\section{Material e Métodos}

\section{Cultivo dos animais}

O ambiente experimental foi instalado na Associação de Pescadores da praia da Penha, no litoral sul do município de João Pessoa, Paraíba. O experimento foi realizado nos meses de setembro a novembro do ano de 2015.

Neste local foram instaladas oito caixas d'água de polipropileno, com capacidade de 1.000 litros cada, para o cultivo dos camarões marinhos (Litopenaeus vannamei). Outras duas caixas, com a mesma capacidade, foram utilizadas como filtros (físicos e biológicos, constituídos com pedras, cascalhos e folhas de plásticos para o desenvolvimento do perifíton), para a manutenção da qualidade da água. Os tanques foram divididos em dois circuitos, de quatro tanques com um filtro cada.

As pós-larvas dos camarões Litopenaeus vannamei foram adquiridas da empresa Tecmares Ltda, no município de Canguaretama, RN, com uma idade PL 12. Cada caixa d'água dos circuitos, com exceção daquelas com os filtros, receberam no total 120 larvas de camarões que foram cultivados durante 94 dias. Antes de iniciar a oferta das rações, os indivíduos, de ambos os circuitos, foram alimentados duas vezes ao dia durante duas semanas com zooplâncton e fitoplâncton, por estimulação biológica aplicando um biocomposto obtido a partir da mistura de 
água do mar e resíduos de frutas e legumes. Após esta etapa, os animais foram alimentados com ração industrial e ração doméstica, produzida pelos pescadores da Associação, segundo metodologia de Oliveira e Crispim (2013), durante todo o experimento.

Os animais do cultivo Tratamento Doméstico (TD) receberam ainda uma porção diária de zooplâncton e fitoplâncton, até 60 dias após início do cultivo. A quantidade de ração atribuída foi a mesma em ambos os ensaios e na proporção de $2 \mathrm{~g}$ nos primeiros 30 dias e $4 \mathrm{~g}$ nos 60 dias seguintes, sempre ofertados duas vezes ao dia.

A água utilizada no bioensaio consistiu numa mistura de água do mar com água doce, proveniente da rede de abastecimento local, na proporção de 1:1, e salinidade entre 0 e 10\%, mantida sob aeração constante. Para oxigenação da água foram utilizadas bombas de aquário, com capacidade de $2000 \mathrm{~L} / \mathrm{h}$, acopladas a canos de policloreto de vinila (PVC) para aspersão da água proveniente do filtro em cada caixa d'água conectada a este. Tal mecanismo contribuiu para a aeração do meio, bem como para a manutenção da circulação da água por entre os tanques do circuito, uma vez que as caixas estavam conectadas ao filtro por meio de mangueiras, mantendo o nível da água equilibrado em todo o circuito experimental.

Como comparativo da eficiência do sistema de perifíton, no ensaio do Tratamento Doméstico (TD) foram instaladas estruturas compostas de plástico e madeira no formato de uma pequena estante, apenas em nas caixas 1 e 2 nomeadas de TD1e TD2, respectivamente. Essas estruturas foram instaladas a fim de comparar o crescimento dos animais nesse circuito. Os animais cultivados em TD1 e TD2 foram comparados quanto ao peso e crescimento com os camarões das caixas TD3 e TD4, do mesmo circuito que não continham as estruturas de perifíton.

\section{Produção da ração doméstica}

A ração doméstica foi produzida a partir dos resíduos de hortifrutigranjeiros, como sobras de cascas de verduras e frutas oriundas das residências dos pescadores e restaurantes instalados na comunidade, o farelo de coco e casca de mandioca, além de músculo e vísceras de bagre-bandeira (Bagre marinus), proveniente das peixarias instaladas no local. Os ingredientes foram separados e armazenados em sacolas plásticas, de uso comercial, estocados em congeladores domésticos, em temperatura de $-18{ }^{\circ} \mathrm{C}$, nas dependências da Associação de Pescadores da Penha, antes de serem processados.

O farelo de côco e a casca de mandioca foram submetidos a um pré-processamento térmico. A casca da mandioca que foi exposta ao sol para secar por cerca de 48 horas. Após esse período, foi triturada uma vez no liquidificador de uso doméstico até obter um pó homogêneo. O farelo do côco foi triturado, retirado o excesso de leite, e posteriormente submetido ao sol para secagem por cerca de 72 horas.

Os resíduos de hortifrutigranjeiros e o bagre foram cortados manualmente em pequenos pedaços, de aproximadamente $5 \mathrm{~cm}$, em seguida foram processados em um moedor de carne de uso comercial, até obter a consistência pastosa. Em seguida foram misturados com o farelo de mandioca e de côco. Após a obtenção da mistura, foram adicionados os compostos químicos fosfato bicálcico $\left(\mathrm{Ca}\left(\mathrm{HPO}_{4}\right) 2 \mathrm{H}_{2} \mathrm{O}\right)$ e um suplemento vitamínico e mineral comercial para carcinicultura. A ração foi novamente levada ao moinho para formação dos grânulos, característicos das rações. Na última fase, as rações foram secas em estufas de secagem no LABEA em temperatura $50{ }^{\circ} \mathrm{C}$ por 24 horas para retirar completamente a umidade.

\section{Análises bromatológicas das rações e camarões}

As duas rações foram submetidas a análises bromatológicas, para determinação da composição química de macronutrientes, de carboidratos, gordura (extrato etéreo), teores de umidade, cinzas e proteínas. O extrato etéreo foi determinado seguindo os procedimentos descritos por Folch, Less; Stanley (1957). Os carboidratos foram 
determinados por diferença (100 - $\sum$ Umidade + cinzas + gordura + proteína). Elas foram determinadas utilizando a metodologia descrita na AOAC (2000).

Após a despesca dos camarões cultivados no bioensaio, para fins de comparação foram feitas as mesmas análises nas amostras dos três lotes de camarões (dos cultivos, do mercado local e dos camarões industrializados). Após a obtenção destes dados, os mesmos foram confrontados entre si, visando determinar dentre estas amostras qual apresentava maior valor nutricional.

Foram aplicados o teste não-paramétrico de Wilcoxon-Mann-Whitney e o teste paramétrico t-Student e os testes de normalidade de Lilliefors, Shapiro-Wilk e Anderson-Darling nos dados obtidos na biometria dos animais. Nos dados obtidos das análises bromatológicas na ração doméstica e industrial, bem como nas bromatológicas nos camarões do bioensaio e nos camarões comercializados na região, foram avaliados a média dos resultados e o percentual de composição dos macronutrientes, cinzas e umidade

\section{Resultados e Discussão}

\section{Cultivo dos animais}

Após o período de cultivo de 94 dias, os animais foram despescados e retirados 20 espécimes de cada tanque, exceto TI1, a fim de proceder à biometria dos animais. Os dados apontam que em ambos os cultivos os indivíduos apresentaram crescimento e peso semelhantes, sendo 8,2cm de comprimento e peso de 3,6g em média.

Quanto à comparação dos animais cultivados no TD, nos tanques contendo as estruturas promotoras com perifítons (TD1 e TD2), constatou-se que os camarões cresceram mais que os animais cultivados nos outros dois tanques, com média de comprimento de $8,42 \mathrm{~cm}$ e peso de 4,02g. Nos tanques TD3 e TD4, a média de comprimento foi de $7,96 \mathrm{~cm}$ e peso de $3,2 \mathrm{~g}$.

Servilla et al. (2004), relataram resultados inferiores, em cultivo intensivo de camarões marinhos em tanques com circulação parcial de água, com animais de peso médio de 4,0 g, com variação mínima de 0,4g e no máximo 8,5g respectivamente em um cultivo de 168 dias, ou seja, mais 74 dias que o deste estudo. Em seu trabalho, Krummenauer et al. (2012) avaliou o cultivo de camarões em sistema de bioflocos (Biofloc Tecnology System) comparando tratamentos com 2,5, 10 e 100\% de reutilização de água, obtiveram um peso médio final de 2,91, 3,25 e 3,46g, respectivamente. Isso demonstra que a ração doméstica produzida foi eficiente no crescimento do camarão para o período de cultivo de 94 dias.

Estatisticamente, através do teste paramétrico t-Student ( $p$-valor $<0,05)$, conclui-se que existe diferença significativa entre os grupos (com biofilme) e (sem biofilme) ao nível de $5 \%$ ( $p$-valor $<0,05$ ), o que demonstra que a presença do biofilme foi um fator relevante no cultivo dos camarões cultivados em TD1 e TD2. O alimento fornecido pela presença do biofilme adicional contribuiu para o aumento no peso destes organismos, mas é possível que a qualidade de água também tivesse sido melhorada, diminuindo a situação de estresse nessas caixas de água.

Tais resultados mostram que a presença dos perifítons nos biofilmes pode ter contribuído para a redução dos níveis dos substratos metabólicos produzidos pelos camarões, atuando na reciclagem dos nutrientes e na conversão alimentar destes, tornando-os uma fonte extra de alimentos, em forma de algas, e como alimentos outros micro-organismos presentes nos zooplâncton e fitoplâncton consumidos pelos camarões, aumentando assim seu consumo alimentar no cultivo (Granja Russo 2008; Silva 2006). 
A composição das rações utilizadas no bioensaio foram determinantes para os resultados encontrados. A comparação entre a ração industrial (RI) e ração doméstica $(\mathrm{RD})$ nos lotes 1 e 2, aponta diferentes constituições dos macronutrientes, principalmente em relação à proteína, lipídios e umidade, onde RI apresenta maiores concentrações. Seguindo esse comportamento, RI apresenta percentuais de cinzas maiores no lote 1, e uma redução, comparado a RD, no segundo lote. Já em relação aos carboidratos, RD mostra teor maior que RI, em ambos os lotes.

Os resultados bromatológicos mostram que ambas as rações utilizadas no bioensaio possuíam uma prevalência de proteína em proporções distintas, para RI (48,29 e 44,3 \%) e para RD (37,78 e 18,64\%), nos lotes 1 e 2 , respectivamente, conforme aponta dados na Tabela 1. Segundo SEBRAE (2005), os teores de proteína adequados na ração para alimento de camarões, é entre 25 e 30\%, o que a ração doméstica atendeu esta exigência apenas no primeiro lote. Essa diferença, no entanto, não se refletiu no crescimento dos indivíduos que foi semelhante nos dois tratamentos.

Tabela 1 - Dados bromatológicos das rações industrial e doméstica

\begin{tabular}{ccccc}
\hline $\begin{array}{c}\text { Composição } \\
\%\end{array}$ & $\begin{array}{c}\text { Ração Industrial } \\
\text { Lote 1 }\end{array}$ & $\begin{array}{c}\text { Ração Industrial } \\
\text { Lote 2 }\end{array}$ & $\begin{array}{c}\text { Ração Doméstica } \\
\text { Lote 1 }\end{array}$ & $\begin{array}{c}\text { Ração Doméstica } \\
\text { Lote 2 }\end{array}$ \\
\hline & MÉDIA & MÉDIA & MÉDIA & MÉDIA \\
Umidade & 9,64 & 12,7 & 2,74 & 1,09 \\
Cinzas & 11,37 & 9,97 & 11,31 & 4,87 \\
Proteína & 48,29 & 44,3 & 37,78 & 18,64 \\
Lipídio & 13,57 & 12,25 & 6,48 & 5,45 \\
Carboidrato & 17,13 & 20,78 & 41,69 & 69,95 \\
\hline
\end{tabular}

Dentre os macronutrientes ofertados aos camarões, podemos destacar a proteína como uma das mais importantes na dieta alimentar, pois a sua oferta está diretamente relacionada ao fornecimento de aminoácidos indispensáveis para o desenvolvimento animal, que por serem onívoros buscam suas requisições com uma dieta mista, de origem animal e vegetal (Pontes et al. 2010). Dada sua importância, e o componente mais caro das rações comerciais. Entretanto, a ausência de publicações e normas técnicas que discipline as exigências nutricionais dos camarões cultivados em viveiros, bem como a composição das dietas comerciais formuladas adequadas, tem contribuído para a produção de rações com excesso de nutrientes, especialmente proteína.

Neste sentido, Tacon (2002) recomenda que a composição das rações comerciais deve buscar outras fontes de proteína e nutrientes como forma de alcançar o desenvolvimento sustentável da carcinicultura, devendo introduzir microorganismos, como fonte de nutrientes na dieta para o camarão cultivado, contribuindo ainda para a manutenção da produtividade, estabilidade e saúde dos sistemas de produção de camarão. Isso porque a suplementação excessiva de proteínas nas rações pode contribuir para sua excreção pelos animais, acumulando-se em forma de amônia e derivados no fundo dos tanques, comprometendo a qualidade da água do cultivo (Costa et al. 2008).

Quanto à umidade, esta foi maior em RI (9,64 e 12,7\%) que em RD (2,74 e 1,09\%) nos dois lotes, respectivamente. SEBRAE (2005) apresenta como teores de umidade adequados em rações de camarões até $10 \%$, o que a ração doméstica atende este critério. Nos lotes 1, de RI e de RD o teor de cinzas foi praticamente o mesmo (RI = 11,37\% e $\mathrm{RD}=11,31 \%)$. No entanto, o segundo lote, de RI (9,97\%) apresentou maior porcentagem do que RD $(4,87 \%)$. Quanto aos lipídios, as maiores concentrações foram encontradas em RI com 13,57 e 12,25\%, e RD apresentou valores de 6,48\% e 5,45\%, nos lotes 1 e 2, respectivamente. Os valores de lipídios na ração industrial (RI) foram mais elevados que os propostos por SEBRAE (2005) que aponta percentuais de 6 a $8 \%$ como os mais adequados. 
Neste nutriente, a ração doméstica (RD) foi mais adequada, com percentuais entre 5 e $6 \%$. Os carboidratos apresentaram quantidade superior nos lotes 1 e 2 em RD (41,69 e 69,95\%), quando comparados com os 1 e 2 lotes de RI (17,13 e 20,78\%). A RD apresentou valores superiores aos propostos por SEBRAE (2005) que são entre 30 e $40 \%$ e a RI inferiores a estes.

Segundos os resultados encontrados, pode-se constatar que as amostras de camarões do TI (78,9\%), TD (80,6\%) e do Mercado Local (77,6\%), apresentaram percentuais elevados de umidade, o que não foi constatado para os camarões Industrializados (74,5\%). Quanto às concentrações de proteína, todas as amostras de camarão mostram teores semelhantes de proteínas, em torno de $20 \%$, sendo ligeiramente menor nos camarões do Mercado Local e do TD. A quantidade de lipídios apresentou pouca variação entre as amostras 1,64; 1,13; 0,71 e 0,72\% para as amostras do Industrializado, e Mercado local, TI e TD, respectivamente. Quanto aos carboidratos as amostras de TI (2,3\%) e TD (1,58 \%) são as que apresentaram maiores concentrações, seguidos do Industrializado (1,42\%) e Mercado local (1,07\%), conforme demonstra os dados na Tabela 2.

Tabela 2 - Composição nutricional dos camarões.

\begin{tabular}{ccccc}
\hline \multirow{2}{*}{ Composição \% } & Camarão Industrializado & $\begin{array}{c}\text { Camarão } \\
\text { Mercado } \\
\text { Local }\end{array}$ & $\begin{array}{c}\text { Camarão Tratamento } \\
\text { Industrial }\end{array}$ & $\begin{array}{c}\text { Camarão } \\
\text { Tratamento } \\
\text { Doméstico }\end{array}$ \\
\hline Umidade & MÉDIA & MÉDIA & MÉDIA & MÉDIA \\
Cinzas & 74,47 & 77,63 & 78,9 & 80,59 \\
Proteína & 2,26 & 1,81 & 1,52 & 1,54 \\
Lipídio & 20,20 & 18,46 & 21,17 & 18,73 \\
Carboidrato & 1,64 & 1,13 & 0,71 & 0,72 \\
\hline
\end{tabular}

Os resultados apontam que os camarões do TD possuem composição nutricional semelhante aos camarões cultivado no TI e aos comercializados no Mercado local e Industrializado. Ao analisar os resultados da composição dos camarões de TD (18,73\% e 0,72\%) dos percentuais de proteína e lipídios respectivamente, pode-se constatar que apresentaram teores de proteína inferiores e de lipídios semelhantes aos animais de TI (21,17\% e 0,71\%), no entanto, os animais desenvolveram-se adequadamente com pequenas variações em média. Esse desempenho em TD pode estar relacionado com a oferta de biofilme com perifíton, que pode ter contribuído no aumento da oferta de proteínas, vitaminas e sais minerais (Moschini-Carlos 1999; Martins Fernandes 2011).

\section{Conclusão}

Os animais cultivados sob TD se desenvolveram de maneira similar aos do TI, indicando que a oferta de ração doméstica, com aproveitamento de resíduos de matéria orgânica, associada à presença de fito e de zooplâncton, é uma alternativa viável. Outro importante contribuinte para alcançar esse resultado pode ter sido a existência de estruturas promotoras do biofilme em dois tanques do TD. Embora o biofilme pudesse se desenvolver nas laterais das caixas, em ambos os tratamentos, a existência das estruturas nos tanques TD1 e TD2 pode ter favorecido o crescimento dos animais, constituindo um incremento nutricional aos camarões.

Quanto às análises bromatológicas das rações, foi possível constatar que a ração industrial tem maior percentual de proteínas, umidade e lipídios em relação à ração doméstica.

A composição nutricional dos camarões do TD, apresenta pequena variação nos percentuais, havendo uma redução nos percentuais das proteínas quando comparados aos do mercado local e aos industrializados. Contudo 
as análises bromatológicas dos animais de ambos tratamentos, apontam que os camarões se desenvolveram adequadamente, com pequena variação nos percentuais.

$\mathrm{Na}$ possibilidade do emprego comercial dessa tecnologia pelos pescadores, será necessário ainda capacitação e orientação voltadas para implementar técnicas de boas práticas de fabricação de todo o processo produtivo de modo a adequar à produção dos animais a legislação sanitária e ambiental vigentes.

Esses resultados apontam que a ração doméstica associada com a oferta regular de biofilmes podem ser utilizados como substitutos totais da ração industrial no cultivo familiar de camarões, pois apresentam bons indicadores de sustentabilidade socioambiental e qualidade nutricional, como o baixo custo na produção, a ausência de aditivos e conservantes, o aproveitamento de resíduos gerados pela comunidade, além da possibilidade de melhoria da qualidade de vida dos pescadores, cuja atividade está cada vez mais ameaçada pelo desenvolvimento da pesca comercial.

O cultivo sustentável desses animais agrega valor comercial ao produto, além de possibilitar a interiorização do cultivo de camarões orgânico nos moldes da produção familiar, uma vez que utiliza espaços físicos disponíveis, inclusive fora das áreas historicamente ocupadas pela atividade. A possibilidade de cultivar camarão fora desses ambientes favorece a gestão dos recursos naturais, uma vez que reduz o consumo de água, a geração de efluentes, favorece a geração de emprego e renda para grupos tradicionais socialmente excluídos das áreas atualmente ocupadas pelas fazendas de camarão. Isso pode reduzir a dependência da operacionalização da carcinicultura em ecossistemas de mangues, apicuns e áreas de encostas, reduzindo assim os impactos inerentes da atividade da carcinicultura tradicional.

\section{Agradecimentos}

Agradecer o apoio da Coordenação de Aperfeiçoamento de Pessoal de Ensino Superior (CAPES) ao Programa de Pós-graduação de Desenvolvimento e Meio Ambiente vinculado à Universidade Federal da Paraíba, através da atribuição de bolsa ao primeiro autor, e ao MEC/SESu pelo financiamento do projeto de extensão "Aquicultura Familiar como forma de desenvolvimento sustentável".

O presente artigo foi produto da dissertação de Mestrado da primeira autora, publicado como resumo expandido nos anais no XXV Congresso Brasileiro de Ciência e Tecnologia dos Alimentos, e no X Section VI Inrernational Technical Symposium, realizado em outubro de 2016 em Gramado, no Rio Grande do Sul.

\section{REFERÊNCIAS}

Association of Official Analytical Chemists - AOAC. 2000. Official Methods of Analysis. Washington D.C.: AOAC, 1018 p.

Audelo-Naranjo JM, Voltolina D, Romero-Beltrán E. 2012. Culture of white shrimp (Litopenaeus vannamei Boone, 1931) with zero water exchange and no food addition: an eco-friendly approach. Latin American Journal of Aquatic Research 40(2): 441-447.

Alves-Costa FA, Costa RC. 2004. Níveis de metais pesados no camarão-rosa Farfantepenaeus brasiliensis (Crustacea, Decapoda) na enseada de Ubatuba, Ubatuba, São Paulo. Revista de Biociências, Taubaté, v.10, n. 4, p. 199-203, out./dez.

Brasil. 2010. Ministério da Saúde. Secretaria de Vigilância em Saúde. Departamento de Vigilância Epidemiológica. Manual integrado de vigilância, prevenção e controle de doenças transmitidas por alimentos / Ministério da Saúde, Secretaria de 
Vigilância em Saúde, Departamento de Vigilância Epidemiológica. 158 p.: il. (Série A. Normas e Manuais Técnicos), Brasília:

\section{Editora do Ministério da Saúde.}

Brasil. 2003. Ministério da Agricultura Pecuária e Abastecimento. Secretaria de Defesa Agropecuária. Instrução Normativa $\mathrm{N}^{\circ}$ 53, de 2 de Jul de 2003. Dispõe sobre o Regulamento Técnico do Programa Nacional de Sanidade de Animais Aquáticos. Disponível em: http://www.crmvgo.org.br/legislacao/pescado/INM00000053.pdf. Acesso em 16 de out 2014.

Brasil. 2002. Conselho Nacional de Meio Ambiente, Resolução CONAMA nº 312, de 10 de outubro de 2002. Dispõe sobre o licenciamento ambiental dos empreendimentos de carcinicultura na zona costeira. Publicada no DOU n 203 , de 18 de out de 2002, Seção 1, págs. 60-61.

Brasil. 2005. Conselho Nacional de Meio Ambiente, Resolução CONAMA nº 357, de 17 de março de 2005. Alterada pela Resolução 410/2009 e pela 430/2011 Dispõe sobre a classificação dos corpos de água e diretrizes ambientais para o seu enquadramento, bem como estabelece as condições e padrões de lançamento de efluentes, e dá outras providências. Publicada no DOU nº 053, de 18/03/2005, págs. 58-63.

Brasil. 1988. Constituição da República Federativa do Brasil, de 5 de outubro de 1988. Brasília. DF: Senado; 1988. Disponível em http://www.planalto.gov.br/ccivil_03/constituicao/constituicao.htm. Acesso em 11 de jun de 2013.

Costa WM, Gálvez AO, Brito LO, Santos EL. 2008. Produção de ortofosfato, amônia, nitrito e nitrato no cultivo de Litopenaeus vannamei utilizando dietas com diferentes níveis de proteína vegetal e animal. Boletim do Instituto de Pesca, 34(2), $303-310$.

Companhia Ambiental do Estado de São Paulo - CETESB. 2007. Norma Técnica L5.406 Coliformes termotolerantes: Determinação em amostras ambientais pela técnica de tubos múltiplos com meio A1 - método de ensaio. Jun.

Companhia Ambiental do Estado de São Paulo - CETESB. 2014. Norma Técnica L5.306 $3^{a} 14$ páginas. Determinação de Clorofila a e Feofitina a: método espectrofotométrico. Edição Fev.

Capistrano Sobrinho D. Estudo do crescimento, estabilidade física, química e termogravimétrica com rações para camarões marinho Litopeneaus vannamei. Dissertação (Mestrado). Tecnologia em Alimentos. Universidade Federal da Paraíba, 2011.

Coutinho DA. 2008. Aspectos da toxicocinética do mercúrio em camarões de cultivo Litopeneaus vannamei. Dissertação (Mestrado). Ciências Biológicas (Biofísica). Universidade Federal do Rio de Janeiro.

Dias HM, Soares MLG, Neffa E. 2012. Conflitos Socioambientais: O caso da carcinicultura no complexo estuarino Caravelas - nova Viçosa/Bahia-Brasil. Ambiente \& Sociedade. São Paulo v. XV, n. 1 _ p. 111-130 - jan.-abr.

Folch J, Less M, Stanley SA. 1957. Simple method for the isolation and purification of total lipids from animal tissues. Journal of Biological Chemistry, 226, 497-509.

Figuerêdo MCB, Araújo LFP, Freitas Rosa M, Morais LFS, Paulino WD, Gomes RB. 2006. Impactos Ambientais da Carcinicultura de Águas Interiores. Engenharia Sanitária e Ambiental. 231s. Vol.11 - No 3 - jul/set, 231-240.

Furtado PS, Borba MR, Maicá PF, Wasielesky Junior W. 2010. Suplementação de taurina em dietas com duas concentrações proteicas para pós-larvas de camarão-branco-do-pacífico. Revista Brasileira de Zootecnia, v.39, n.11, p.2330-2335.

Kasper D, Botaro D, Palermo EFA, Malm O. 2007. Mercúrio em Peixes - Fontes e Contaminação. Ecologia Brasileira, 11 (2) 228-239. 
Krummenauer D, Seifert-Júnior CA, Poersch LH, Foes GK, De Lara GR, Wasielesky-Junior W. 2012. Cultivo de camarões marinhos em sistema de bioflocos: análise da reutilização da água. Atlântica, Rio Grande, 34(2), 103-111.

Martins FCO, Fernandes VO. 2011. Biomassa e Composição elementar (C, N e P) perifítica no alto rio de Santa Maria da Vitória, Espirito Santo, Brasil. Brazilian Jornal of Aquatic Science and Technology, 15(1), 11-18.

Moschini-Carlos V. 1999. Importância, estrutura e dinâmica da comunidade perifídica nos ecossistemas aquáticos continentais. Pompêo, M. L. M. (Ed.) Perspectivas na Limnologia do Brasil, pp. 91-103. Capítulo 6. Disponível em http://www.ib.usp.br/ limnologia/Perspectivas/arquivo\%20pdf/Capitulo\%206.pdf. Acesso em 28/09/2014.

Oliveira FMF, Crispim MCB. 2013. Aquicultura sustentável como forma alternativa de minimizar os impactos desta produção em comunidades pesqueiras. Gaia Scientia, 7(1): 64-79.

Pontes CS, Santos DB, Bessa Júnior AP, Arruda AMV. 2010. Substituição de ração no crescimento de juvenis do camarão marinho Litopenaeus vannamei em laboratório. Revista Caatinga, Mossoró, v. 23, n. 1, p. 121-126, jan.-mar.

Serviço Brasileiro de Apoio às Micro e Pequenas Empresas - SEBRAE. 2008. Aqüicultura e pesca: camarões. Relatório Completo. Estudo de Mercado SEBRAE/ESPM, set.

Serviço de apoio às micro e pequenas empresas do Espírito Santo- SEBRAE/ES. 2005. Tecnologia de criação do camarão da Malásia (Macrobracbium rosenbergii) Manual de Carcinicultura de Água Doce. Centro de Tecnologia em Aqüicultura e Meio Ambiente LTDA- CTA, Vitória.

Sevilla BB, Ramirez LFB, Rodriguez MH. 2004. Intensive Culture of Litopenaens venamei Boone, 1931, in s recirculating seawater system. Ciências Marinas. Marzo ano vol. 30, número 1B. Universidad Autónoma de Baja California. Enseada, Mexico. pp 179-188.

Silva CF. 2006. Análise da qualidade nutricional do biofilme. Dissertação (Mestrado) Aquicultura. Fundação Universidade Federal do Rio Grande do Sul, Rio Grande.

Rolim NPFA. 2015. Produção familiar do camarão Litopenaeus vannamei (Boone, 1931): viabilidade e qualidade. Dissertação (Mestrado). Centro de Ciência Exatas e da Natureza, Universidade Federal da Paraíba.

Tacon AGJ. 2002. Thematic review of feeds and feed management practices in shrimp aquaculture. Report prepared under the World Bank, NACA, WWF and FAO 86. Consortium Program on Shrimp Farming and the Environment. Work in Progress for Public Discussion. Kaneohe, HI, USA: Consortium, 69p.

United Nations Food and Agriculture - FAO. 2006. International Principles for Responsible Shrimp Farming. Network of Aquaculture Centres in Asia-Pacific (NACA). Bangkok, Thailand. 20 pp. FAO/NACA/UNEP/WB/WWF. Disponível em <http://www.pnuma.org.br/admin/publicacoes/texto/Principios_Internacionais_Carcinicultura_Responsavel.pdf.> Acesso em 10 de dez de 2013. 